UTCCP-P-94

Oct. 2000

\title{
Full QCD Light Hadron Spectrum and Quark Masses: Final Results from CP-PACS*
}

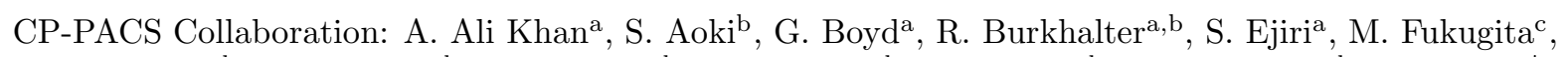

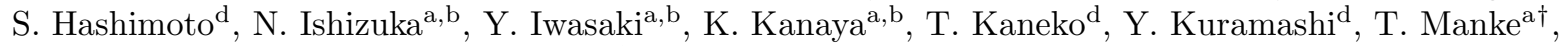
K. Nagai ${ }^{\mathrm{a}}$, M. Okawa ${ }^{\mathrm{d}}$, H.P. Shanahan ${ }^{\mathrm{a}}$, A. Ukawa ${ }^{\mathrm{a}, \mathrm{b}}$ and T. Yoshiéa,b

${ }^{a}$ Center for Computational Physics, University of Tsukuba, Tsukuba, Ibaraki 305-8577, Japan

${ }^{\mathrm{b}}$ Institute of Physics, University of Tsukuba, Tsukuba, Ibaraki 305-8571, Japan

${ }^{\mathrm{C}}$ Institute for Cosmic Ray Research, University of Tokyo, Kashiwa 277-8582, Japan

${ }^{\mathrm{d} H i g h}$ Energy Accelerator Research Organization (KEK), Tsukuba, Ibaraki 305-0801, Japan

We present the final results of the CP-PACS calculation of the light hadron spectrum and quark masses with two flavors of dynamical quarks. Simulations are made with a renormalization-group improved gauge action and a mean-field improved clover quark action for sea quark masses corresponding to $m_{\mathrm{PS}} / m_{\mathrm{V}} \approx 0.8-0.6$ and the lattice spacing $a=0.22-0.11 \mathrm{fm}$. For the meson spectrum in the continuum limit a clearly improved agreement with experiment is observed compared to the quenched case, demonstrating the importance of sea quark effects. For light quark masses we obtain $m_{u d}^{\overline{M S}}(2 \mathrm{GeV})=3.44_{-0.22}^{+0.14} \mathrm{MeV}$ and $m_{s}^{\overline{M S}}(2 \mathrm{GeV})=88_{-6}^{+4} \mathrm{MeV}(K$-input) and $m_{s}^{\overline{M S}}(2 \mathrm{GeV})=90_{-11}^{+5} \mathrm{MeV}$ ( $\phi$-input), which are reduced by about $25 \%$ compared to the values in quenched QCD.

\section{INTRODUCTION}

Recent progress in lattice calculations of the quenched light hadron spectrum [1] has demonstrated clearly that effects of dynamical sea quarks have to be included for a precision comparison with the experimental spectrum. As a first step towards the real world we have pursued a systematic calculation in two-flavor QCD over the last 3 years [2,3]. In this article we present a summary of the final results on the light hadron spectrum and quark masses from this calculation.

In order to deal with the increased demand in computer time for full QCD we have used an RGimproved gauge action and a tadpole-improved SW clover quark action, which allows us to simulate at coarse lattices in the range $a=0.22-0.11$ fm [2]3]. Since Lattice'99 [3] we have doubled the statistics at the smallest lattice spacing on a $24^{3} \times 48$ lattice, with which we have reached

\footnotetext{
*talk presented by R. Burkhalter

†address after 1 Feb., 2000: Department of Physics, Columbia University, $538 \mathrm{~W}$ 120th St., New York, NY 10027, USA

$\ddagger$ address after 15 Sept., 2000: Department of Biochemistry and Molecular Biology, University College London, London, England, UK
}

our planned target of three lattice spacings, each with a similar amount of statistics at each simulated sea quark mass. The final parameters of our simulations are summarized in Table 1 .

We have also made a set of simulations in quenched QCD using the same improved actions in the same range of lattice spacings and with a similar range of quark masses. This allows us to compare in detail spectrum results in full and quenched QCD and to make an independent confirmation of the findings in quenched QCD in Ref. [1]. Parameters of quenched simulations are summarized in Table 2 .

\section{SEA QUARK EFFECTS IN THE LIGHT HADRON SPECTRUM}

A novel feature in the spectrum analysis with two-flavor QCD is the dependence of hadron masses on both the sea and the valence quark mass. We take this into account by a combined quadratic ansatz. For vector mesons and decuplet baryons the formula has the form,

$$
\begin{aligned}
m_{V, D}= & A+B_{s} \mu_{\text {sea }}+B_{v} \mu_{v a l} \\
& +C_{s} \mu_{\text {sea }}^{2}+C_{v} \mu_{v a l}^{2}+C_{s v} \mu_{\text {sea }} \mu_{v a l}
\end{aligned}
$$


Table 1

Parameters and final statistics of two-flavor QCD simulations.

\begin{tabular}{ccccccccc}
\hline$\beta$ & $L^{3} \times T$ & $c_{S W}$ & $a[\mathrm{fm}]$ & $L a[\mathrm{fm}]$ & \multicolumn{3}{c}{$m_{\mathrm{PS}} / m_{\mathrm{V}}$ for sea quarks : \# HMC trajectories } \\
\hline 1.80 & $12^{3} \times 24$ & 1.60 & $0.215(2)$ & $2.58(3)$ & $0.807(1): 6250$ & $0.753(1): 5000$ & $0.694(2): 7000$ & $0.547(4): 5250$ \\
1.95 & $16^{3} \times 32$ & 1.53 & $0.155(2)$ & $2.48(3)$ & $0.804(1): 7000$ & $0.752(1): 7000$ & $0.690(1): 7000$ & $0.582(3): 5000$ \\
2.10 & $24^{3} \times 48$ & 1.47 & $0.108(1)$ & $2.58(3)$ & $0.806(1): 4000$ & $0.755(2): 4000$ & $0.691(3): 4000$ & $0.576(3): 4000$ \\
\hline
\end{tabular}

where $\mu_{v a l}$ represents the mass squared $m_{P S}^{2}$ of a pseudo scalar meson made of a valence quarkantiquark pair and $\mu_{\text {sea }}$ stands for a $P S$ meson made of sea quarks. Since octet baryon masses are not functions of the average valence quark mass we use a more complicated formula with 12 parameters. For quenched results with improved actions terms in $\mu_{\text {sea }}$ are dropped and we find that vector meson and decuplet baryon masses are well described by a linear ansatz in $\mu_{v a l}$. Physical masses in full QCD are obtained from Eq. 11 at $\mu_{\text {sea }}=\mu_{u d}$ and $\mu_{\text {val }}=\mu_{u d}$ or $\mu_{s}$, which are fixed using $\pi, \rho$ and either $K$ or $\phi$ meson masses as input.

Since our simulations with improved action employ a mean-field clover coefficient $c_{S W}=P^{-3 / 4}$ for the quark action, the leading scaling violation is $O\left(g^{2} a\right)$. Here $g^{2}$ is the renormalized coupling $g_{\overline{M S}}^{2}(\mu)$ 柾, evaluated at a fixed scale $\mu$. Higher order contributions consist of terms of $O\left(g^{4} a \log (a)\right), O\left(a^{2}\right)$ etc. However, the number and range of lattice spacings restrict our choice of fitting form to the leading scaling violation term, linear in $a$, which we apply for extrapolations to the continuum limit both in quenched and full QCD.

Our main results for the meson spectrum are given in Fig. 11 where $\phi$ and $K^{*}$ meson masses obtained with $K$-input are plotted. The new

Table 2

Parameters of quenched QCD simulations.

\begin{tabular}{|c|c|c|c|c|c|}
\hline \multicolumn{3}{|c|}{$\overline{16^{3} \times 32}$} & \multicolumn{3}{|c|}{$24^{3} \times 48$} \\
\hline $\bar{\beta}$ & $a[\mathrm{fm}]$ & $L a[\mathrm{fm}]$ & $\bar{\beta}$ & $a[\mathrm{fm}]$ & $L a[\mathrm{fm}]$ \\
\hline 2.187 & $0.200(2)$ & $3.21(3)$ & $\overline{2.416}$ & $0.145(2)$ & $3.47(4)$ \\
\hline 2.214 & $0.190(2)$ & 3.05 & 2.456 & $0.133(1)$ & $3.19(3)$ \\
\hline 2.247 & 0.1 & $2.89(3)$ & 2.487 & $0.128(1)$ & $3.08(3)$ \\
\hline 2.281 & 0.17 & $2.82(3)$ & 2.528 & $0.121(1)$ & $2.89(3)$ \\
\hline 2.334 & $0.163(2)$ & $2.61(3)$ & 2.575 & $0.113(1)$ & $2.71(3)$ \\
\hline
\end{tabular}

quenched results with the improved action nicely confirm the finding with the standard action in Ref. [1] that the quenched QCD prediction for the $\phi$ and $K^{*}$ masses is smaller by about $4 \%$ than experiment. On the other hand, the two-flavor results exhibit a larger slope than those of quenched $\mathrm{QCD}$, and the values linearly extrapolated to the continuum limit lie within $1 \%$ of the experimental values.

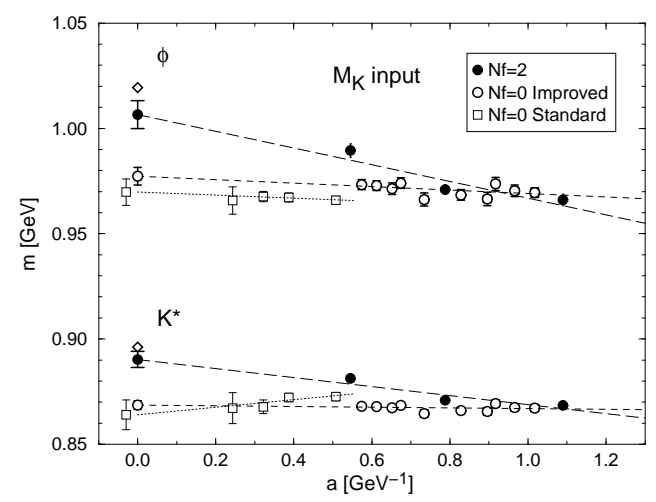

Figure 1. Meson masses in two-flavor (filled symbols) and quenched (open symbols) QCD.

We find this result to be a clear evidence of the importance of sea quark effects. The observed trend that the meson hyperfine splitting is enlarged by such effects is consistent with a qualitative view that the spin-spin coupling in quenched QCD is reduced compared to full QCD due to a faster running of the quenched QCD coupling constant with scale.

Our baryon mass results are collected in Fig. 2. Starting with quenched QCD, our new results with the improved action show sizable statistical fluctuations. With ten points of data, however, one observes a trend that the magnitude of scaling violation is smaller than for the standard action. Linearly extrapolating to the continuum limit, the spectrum for octet and decuplet 


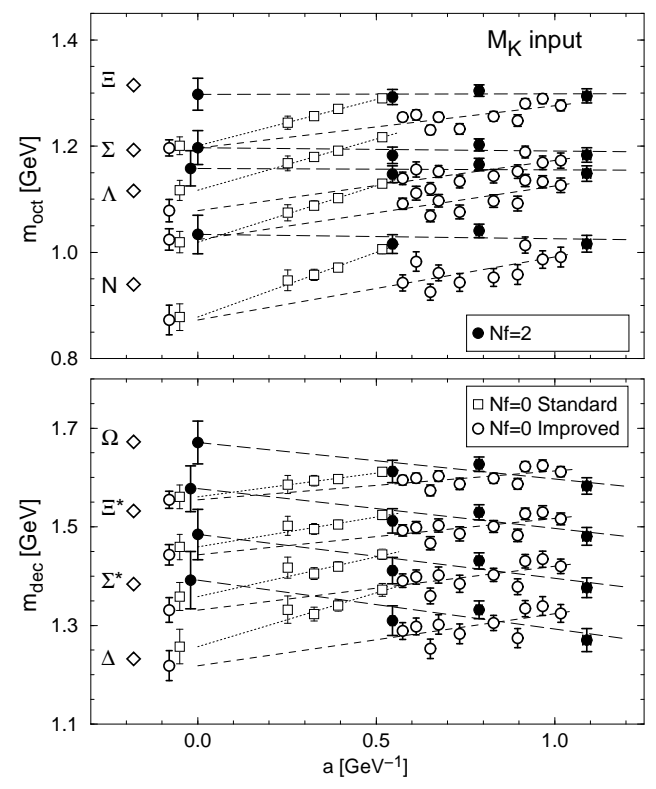

Figure 2. Corresponding plot for baryon masses.

baryons is consistent between the two actions.

A worry with the baryon spectrum is possible finite-size effects. As we see from Table 2 , however, the spatial size mostly lies in the range $L a \approx 2.8-3.5 \mathrm{fm}$, and only in two cases goes down to $L a \approx 2.7-2.6 \mathrm{fm}$. Finite-size effects are suppressed in quenched QCD due to $Z(3)$ symmetry[5]. Past studies $[6]$ also indicate that $L a \approx 3 \mathrm{fm}$ is sufficient to contain the magnitude of finite-size effects for baryons within a few \% level. We therefore consider that our quenched baryon results do no suffer seriously from finitesize contaminations.

Clear conclusions are difficult to draw for twoflavor results. Since quenched results are generally smaller than experiment, we expect sea quark effects to push up the baryon masses. While this is a general trend observed in our data, the nucleon and the $\Delta$ masses turn out higher than experiment, whereas a better agreement is seen for heavier strange baryons.

Finite-size effects are severer in full QCD with dynamical sea quarks since suppression due to $Z(3)$ symmetry is no longer effective. It is probable that the spatial size of $2.5 \mathrm{fm}$ in full QCD, which is smaller than the smallest quenched lattice, is too small to avoid finite-size effects for
Table 3

Final results for quark masses in $\overline{M S}$ scheme at $\mu=2 \mathrm{GeV}$ (in $\mathrm{MeV}$ ).

\begin{tabular}{lcccc}
\hline & action & $m_{u d}$ & \multicolumn{2}{c}{$m_{s}$} \\
& & & $K$-input & $\phi$-input \\
\hline$N_{f}=2$ & impr. & $3.44_{-0.22}^{+0.14}$ & $88_{-6}^{+4}$ & $90_{-11}^{+5}$ \\
$N_{f}=0$ & impr. & $4.36_{-0.17}^{+0.14}$ & $110_{-4}^{+3}$ & $132_{-6}^{+4}$ \\
$N_{f}=0$ & stand.[1] & $4.57(18)$ & $116(3)$ & $144(6)$ \\
\hline
\end{tabular}

baryons, especially those made of light up and down quarks. Further studies on larger spatial sizes are needed to convincingly demonstrate sea quark effects in the baryon mass spectrum.

\section{LIGHT QUARK MASSES}

The light quark masses are fundamental parameters of Nature which can only be determined by hadron spectrum calculations. In Table 3 we present our two-flavor results for up-down and strange quark masses in the $\overline{M S}$ scheme at $\mu=$ $2 \mathrm{GeV}$, and compare them with quenched results. These results have been published in Ref. [7]. The quenched results, which are consistent between the two types of actions, overestimate the quark mass by about $25 \%$ compared to two-flavor QCD. Furthermore, a discrepancy in the strange quark mass in quenched QCD depending on the experimental input is reduced to a level well contained in the error of about $10 \%$. Both these features demonstrate a significant improvement in the determination of light quark masses through inclusion of two flavors of dynamical quarks.

Our results are obtained from analyses of two definitions of quark mass, one derived from the axial Ward identity $\nabla_{\mu} A_{\mu}(x)=2 a m_{q}^{\mathrm{AWI}} P(x)$, and another $m_{q}^{\mathrm{VWI}}=\left(1 / \kappa-1 / \kappa_{c}\right) / 2 a$ derived from the vector Ward identity. For the latter, two choices for $\kappa_{c}$, either the one in full QCD or in partially quenched chiral limit, are examined. In Fig. 3 we illustrate the continuum extrapolation for the strange quark mass. Lines show a linear fit assuming a common value in the continuum limit, which we take to be our central value.

A crucial point is the estimation of systematic errors, which arise from (i) chiral extrapolation, (ii) renormalization factor, and (iii) continuum 


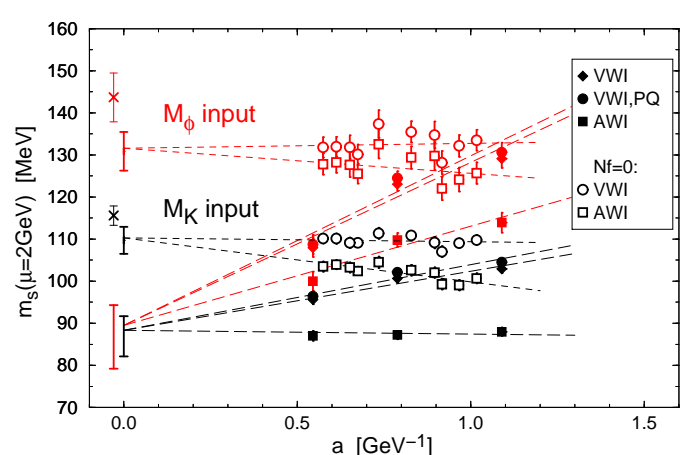

Figure 3. Continuum extrapolation of strange quark mass for improved actions. Results from standard action in Ref.[1] are also shown (crosses).

extrapolation. We estimate the error from (iii) by making linear fits separately to each definition of quark mass and taking the spread of results. The error due to chiral extrapolation (ii) is estimated by replacing quadratic terms in Eq. 1 by terms with power $3 / 2$ which also appear in chiral perturbation theory and by introducing cubic terms in fits of pseudo scalar mesons.

For the renormalization factors relating the lattice quark mass to the $\overline{M S}$ mass in the continuum, we employ the perturbative values at one loop given by $Z_{m}=1+0.0400 g_{\frac{2}{M S}}(\mu)$ for VWI quark mass and $Z_{A} / Z_{P}=1+$ $0.0308 g \frac{2}{M S}(\mu)$ for AWI quark mass. These expressions show that the magnitude of one-loop corrections is about $10 \%$. One loop corrections in improvement coefficients have a similar size. To estimate further corrections from higher order terms, we compare results for two definitions of the mean-field improved coupling constant: $\quad g_{\frac{-2}{M S}}(1 / a)=\left(3.648 W_{1 \times 1}-\right.$ $\left.2.648 W_{1 \times 2}\right) \beta / 6-0.1006+0.03149 N_{f}$, appropri-

Table 4

Contributions to total error in continuum limit.

\begin{tabular}{ccccc}
\hline & stat. & chiral & Z-factor & cont.ext. \\
\hline$m_{u d}$ & $+2.6 \%$ & $+1.2 \%$ & $+2.3 \%$ & $+1.7 \%$ \\
& $-2.6 \%$ & $-2.3 \%$ & $-5.0 \%$ & $-2.3 \%$ \\
$m_{s}(K$-input $)$ & $+2.4 \%$ & $+1.6 \%$ & $+2.2 \%$ & $+1.4 \%$ \\
& $-2.4 \%$ & $-2.2 \%$ & $-5.6 \%$ & $-2.8 \%$ \\
$m_{s}(\phi$-input $)$ & $+4.8 \%$ & $+1.5 \%$ & $+1.7 \%$ & $+0.9 \%$ \\
& $-4.8 \%$ & $-7.6 \%$ & $-6.9 \%$ & $-1.6 \%$ \\
\hline
\end{tabular}

ate for the RG-improved gluon action and alternatively $g_{\frac{-2}{M S}}(1 / a)=W_{1 \times 1} \beta / 6+0.2402+$ $0.03149 N_{f}$. For the matching scale we use the two values $\mu=1 / a$ and $\mu=\pi / a$.

The systematic errors estimated as above are listed in Table 4 . We calculate the total error by adding them by quadrature.

\section{CONCLUSIONS}

Our first systematic study of $N_{f}=2$ full QCD clearly showed the existence of sea quark effects in the meson spectrum and quark masses. Further progress can be expected in the near future from calculations with larger lattices, smaller quark masses and, most importantly, inclusion of dynamical strange quark.

This work is supported in part by Grants-inAid of the Ministry of Education (Nos. 10640246, 10640248, 10740107, 11640250, 11640294, 11740162, 12014202, 12304011, 12640253, 12740133). AAK and TM are supported by JSPS Research for the Future Program (No. JSPSRFTF 97P01102). SE, TK, KN and HPS are JSPS Research Fellows.

\section{REFERENCES}

1. S. Aoki et al. (CP-PACS Collaboration), Phys. Rev. Lett. 84, 238(2000); Nucl. Phys. B(Proc. Suppl.)73, 189(1999).

2. R. Burkhalter, Nucl. Phys. B (Proc. Suppl.) 73, 3(1999); S. Aoki et al. (CP-PACS Collaboration), ibid. 73, 192(1999).

3. A. Ali Khan et al. (CP-PACS Collaboration), Nucl. Phys. B (Proc. Suppl.) 83-84, 176(2000).

4. M. Lüscher et al., Nucl. Phys. B384, 168(1992); ibid. B389, 247(1993).

5. S. Aoki et al., Phys. Rev. D50, 486 (1994) and earlier references therein.

6. See, e.g., MILC Collaboration, C. Bernard et al., Nucl. Phys. B (Proc. Suppl.) 60A, 3 (1998).

7. A. Ali Khan et al. (CP-PACS Collaboration), Phys. Rev. Lett., in print (hep-lat/0004010).

8. S. Aoki et al., Phys. Rev. D 58, 074505 (1998); Y. Taniguchi and A. Ukawa, ibid. 58, 114503 (1998). 